\title{
Combining X-ray diffraction, X-ray absorption spectroscopy, and molecular dynamics simulations to probe metals in zeolites: the case of intergrown $\mathrm{Cd}^{2+}$-LEV/ERI
}

\author{
Georgia Cametti ${ }^{1}$, Andreas C. Scheinost ${ }^{2,3}$, Sergey, V. Churakov ${ }^{1,4}$ \\ ${ }^{1}$ Institute of Geological Sciences, Baltzerstrasse 1+3, 3012, Bern, Switzerland, ${ }^{2}$ The Rossendorf Beamline at the European \\ Synchrotron Radiation Facility (ESRF), Avenue des Martyrs 71, 38043, Grenoble, France, ${ }^{3}$ Helmholtz Zentrum Dresden Rossendorf, \\ Institute of Resource Ecology, Bautzner Landstrasse 400, 01328, Dresden, Germany, ${ }^{4}$ Paul Scherrer Institut, Forschungstrasse 111, \\ 5232, Villingen PSI, Switzerland \\ georgia.cametti@geo.unibe.ch
}

Despite cadmium being a toxic element for environmental and human health, it is widely used in industries for fabrication of nickelcadmium batteries, as anticorrosive agent, color pigment, etc. The most common and effective techniques for Cd removal from wastewater include filtration, chemical precipitation, bio-remediation and ion exchange [1]. Because of their microporous structure and extremely efficient cation exchange capacity, natural zeolites are good candidates for use as ionic filters. Moreover, heavy-metal exchanged zeolites show improved catalytic properties that can be exploited in post remediation processes [2,3]. Therefore, the chemical reactivity and stability of heavy-metal enriched zeolite is of paramount importance. Additionally, the nature of the metal species and their interaction with the zeolite framework play a fundamental role. Nevertheless, the correct determination of the aforementioned aspects can be compromised by the high disorder of the extraframework species, making difficult an unequivocal interpretation of the coordination chemistry of the metal cations. In this respect, the combination of X-ray diffraction (XRD) based techniques together with X-ray absorption spectroscopy (XAS) represents a valid tool to probe the long and short-range order of the species of interest.

In this contribution, we used a complementary experimental and theoretical approach to investigate in detail the structure of two Cd ${ }^{2+}$ exchanged zeolites, levyne (LEV) and erionite (ERI). These two minerals are classified as small-pore zeolites (pore size between 0.35 and $40 \mathrm{~nm}$ ) and, due to their structural similarity, they are often found as intergrown phase in nature [4]. In this study, experimental data from single crystal XRD and XAFS were coupled with Molecular Dynamics (MD) simulations to determine the distribution and coordination chemistry of $\mathrm{Cd}^{2+}$ in the two framework types (LEV and ERI). Our results showed that in Cd-LEV, Cd ${ }^{2+}$ ions have a fairly ordered distribution, resembling that characteristic of the pristine material [5]. In contrast, a strong disorder of the extraframework species $\left(\mathrm{Cd}^{2+}\right.$ and $\left.\mathrm{H}_{2} \mathrm{O}\right)$ is detected in Cd-ERI pores, where the occupancy of the EF sites is lower than $20 \%$. Such disorder was attributed to the presence of $\mathrm{Cd}^{+2}\left(\mathrm{H}_{2} \mathrm{O}\right)_{6}$ complexes, which are only partially coordinated to framework oxygen and, therefore, more mobile. To discriminate between the effect of thermal and structural disorder in the measured and theoretically calculated EXAFS spectra, we propose a theoretical approach based on a set of geometry optimizations performed starting from the uncorrelated atomic configuration of MD simulations [6]. Moreover, based on EXAFS analysis, the formation of metallic Cd within the pores of both zeolites could be ruled out.

Finally, we present the effect of $\mathrm{Cd}^{2+}$ incorporation on the thermal stability of Cd-LEV. The structural changes were monitored in situ from 25 to $400^{\circ} \mathrm{C}$ by single crystal X-ray diffraction. Our results demonstrated that, even if Cd had little influence on the room temperature structure, the dehydration behaviour drastically changes compared to that of the pristine material (natural levyne-Ca). The most relevant differences can be summarized by: i) a stronger volume contraction of the unit-cell volume ( $8 \%$ and $5 \%$ for Cd-LEV and levyne-Ca [5], respectively) in the investigated temperature range, and ii) the lack, at high temperatures, of the phase transformation to levyne B' topology, characteristic of natural levyne-Ca.

[1] Rodriguez-Narvaez, O. M, Peralta-Hernandez, J. M., Goonetilleke, A., Bandala, E. R. (2017). Chem. Eng. J. $323,361$.

[2] Onyestyak, G., Kallo, D. (2003) Microporous and Mesoporous Mater. 61, 199.

[3] Zhang, Y., Qu, Y., Wang, D., Zeng, X. C., Wang, J. (2017) Ind. Eng. Chem. Res. 56, 12508.

[4] Passaglia, E., Galli, E., Rinaldi, R. (1974) Contrib. Mineral. Petrol. 43, 253.

[5] Cametti, G. (2018) Microporous and Mesoporous Mater. 265, 162.

[6] Cametti, G., Scheinost, A. C., Churakov, S. V. (2021) Microporous and Mesoporous Mater. 313, 110835.

Keywords: zeolites; XAFS; XRD; Cd-LEV; Cd-ERI

G. C. acknowledge the Swiss National Science Foundation (SNF) for the Ambizione Grant n. PZ00P2 173997. The access to the Swiss National Supercomputing Center (CSCS) and to UBELIX HPC cluster at the University of Bern is acknowledged. 